\title{
Multi-scale ordering in highly stretchable polymer semiconducting films
}

\author{
Jie Xu® 1,2,11,13, Hung-Chin Wu® 1,13, Chenxin Zhu ${ }^{3}$, Anatol Ehrlich1, Leo Shaw ${ }^{1}$, Mark Nikolka', \\ Sihong Wang1, Francisco Molina-Lopez ${ }^{\mathbb{1} 1}$, Xiaodan Gu ${ }^{114,12}$, Shaochuan Luo ${ }^{5}$, Dongshan Zhou ${ }^{5}$, \\ Yun-Hi Kim ${ }^{6}$, Nathan Ging-Ji Wang ${ }^{1}{ }^{1}$, Kevin Gu1, Vivian Rachel Feig ${ }^{\circledR 7,8}$, Shucheng Chen?, \\ Yeongin Kim ${ }^{3}$, Toru Katsumata ${ }^{1,9}$, Yu-Qing Zheng ${ }^{1}$, He Yan ${ }^{10}$, Jong Won Chung ${ }^{1,10}$, Jeffrey Lopez ${ }^{1}$, \\ Boris Murmann ${ }^{3}{ }^{3}$ and Zhenan Bao ${ }^{1 \star}$
}

Stretchable semiconducting polymers have been developed as a key component to enable skin-like wearable electronics, but their electrical performance must be improved to enable more advanced functionalities. Here, we report a solution processing approach that can achieve multi-scale ordering and alignment of conjugated polymers in stretchable semiconductors to substantially improve their charge carrier mobility. Using solution shearing with a patterned microtrench coating blade, macroscale alignment of conjugated-polymer nanostructures was achieved along the charge transport direction. In conjunction,

영 the nanoscale spatial confinement aligns chain conformation and promotes short-range $\pi-\pi$ ordering, significantly reducing the energetic barrier for charge carrier transport. As a result, the mobilities of stretchable conjugated-polymer films have been enhanced up to threefold and maintained under a strain up to $100 \%$. This method may also serve as the basis for large-area manufacturing of stretchable semiconducting films, as demonstrated by the roll-to-roll coating of metre-scale films.

E ectronics that flex and stretch, behaving much like skin, have been developing quickly and showing potential to significantly impact our daily life ${ }^{1-3}$. Stretchable semiconducting materials are crucial for enabling such electronics. Intrinsic stretchability can provide a number of advantages, including high device density, high data fidelity through near-sensor processing, excellent mechanical robustness and scalable fabrication ${ }^{4}$. Much progress has been made in understanding the structure-property relationships for stretchable materials ${ }^{5,6}$. However, the current state-of-art mobility from stretchable semiconductors can only satisfy the requirements for very limited electronic functionalities? ${ }^{7}$. Approaches that achieve high electrical performance while maintaining good mechanical 2 stretchability remain rare.

Current strategies for high-performance stretchable conjugated polymers are developed mainly on the basis of three categories: (1) tailoring the chemical structure by introducing either soft segments to increase polymer chain flexibility or dynamic interactions to dissipate the applied strain energy ${ }^{8,9},(2)$ increasing the ductility of polymer chains by introducing a nanoconfinement effect to enhance chain dynamics and suppress the growth of large crystallites $^{10,11}$ and (3) engineering the geometry of materials through strain-engineering designs ${ }^{12,13}$. Common among these strategies, the deposition of the polymer films is usually based on spin coating, which tends to give a disordered and isotropic morphology.
However, efficient charge transport favours ordered structures across multiple length scales from the single-chain conformation to the macroscale alignment. At the single-chain level, intrachain transport can be improved by reducing kinks and bending, which can extend $\pi$-electron delocalization along the backbon $e^{14}$. At the nano- to mesoscale, the interchain charge hopping through the overlapping of $\pi$-orbitals can be facilitated by forming short-range ordered polymer aggregates ${ }^{15}$. At the macroscale, the overall charge transport across device channels can be boosted by further aligning polymer chains and aggregations throughout the entire film ${ }^{16}$.

In this work, we develop a solution-shearing method using a blade with line structures, and demonstrate the combination of this solution-shearing method with the nanoconfinement effect (Fig. 1a), so as to achieve both structural ordering across the molecular, mesoand macroscales for simultaneous significantly improved electrical performance and enhanced polymer chain dynamics for mechanical stretchability. Benefiting from multi-scale structural ordering, energetic disorder was reduced, resulting in better $\pi$-electron delocalization and therefore reduced activation energy for charge transport. This method has been applied to the fabrication of stretchable semiconducting films from five different conjugated polymers, which all showed major improvements to their charge carrier mobilities by up to sixfold when compared with their spin-coated (SC) counterparts. The fabricated fully stretchable transistors gave an average initial

'Department of Chemical Engineering, Stanford University, Stanford, CA, USA. ${ }^{2}$ Nanoscience and Technology Division, Argonne National Laboratory, Lemont, IL, USA. 'Department of Electrical Engineering, Stanford University, Stanford, CA, USA. ${ }^{3}$ Stanford Synchrotron Radiation Lightsource, SLAC National Accelerator Laboratory, Menlo Park, CA, USA. ${ }^{5}$ Department of Polymer Science and Engineering, School of Chemistry and Chemical Engineering, Nanjing University, Nanjing, China. ${ }^{6}$ Department of Chemistry and RINS, Gyeongsang National University, Jinju, South Korea. ${ }^{7}$ Department of Chemistry and Hong Kong Branch of Chinese National Engineering Research Center for Tissue Restoration \& Reconstruction, Hong Kong, Hong Kong. ${ }^{8}$ Department of Materials Science and Engineering, Stanford University, Stanford, CA, USA. ${ }^{9}$ Corporate Research and Development, Performance Materials Technology Center, Asahi Kasei Corporation, Shizuoka, Japan. ${ }^{10}$ The Hong Kong University of Science and Technology, Clear Water Bay, Kowloon, Hong Kong. " Present address: Material Research Center, Samsung Advanced Institute of Technology Yeongtong-gu, Suwon-si, South Korea. ${ }^{12}$ Present address: School of Polymer Science and Engineering, University of Southern Mississippi, Hattiesburg, MS, USA. ${ }^{13}$ These authors contributed equally: J. Xu, H.-C. Wu. *e-mail: zbao@@stanford.edu 
a
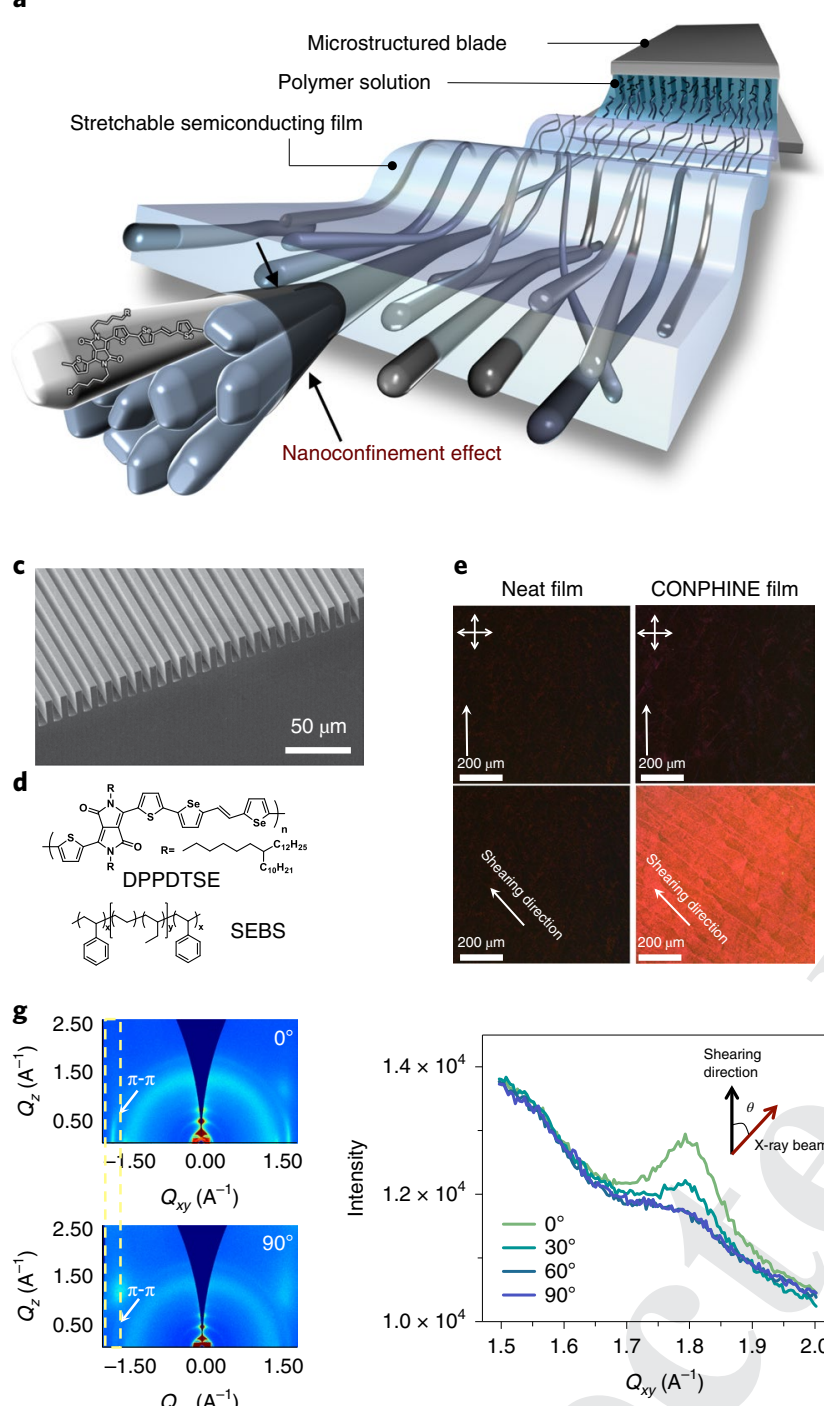

i

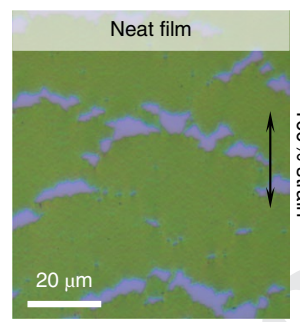

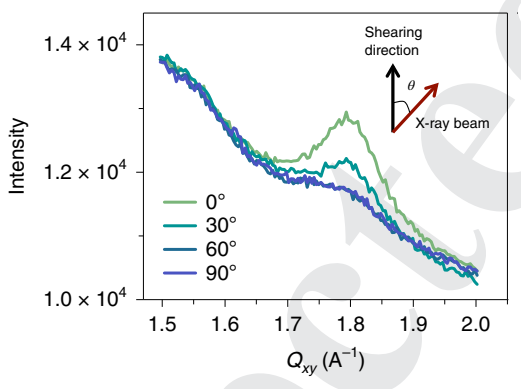

$Q_{x y}\left(\mathrm{~A}^{-1}\right)$ b

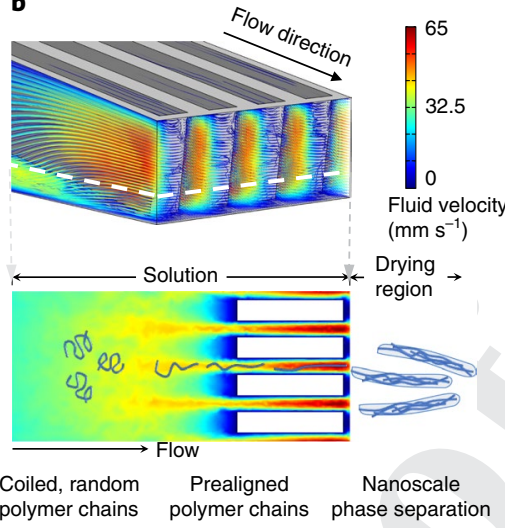

f

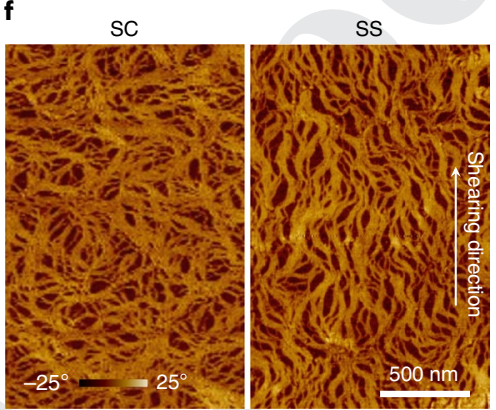

h

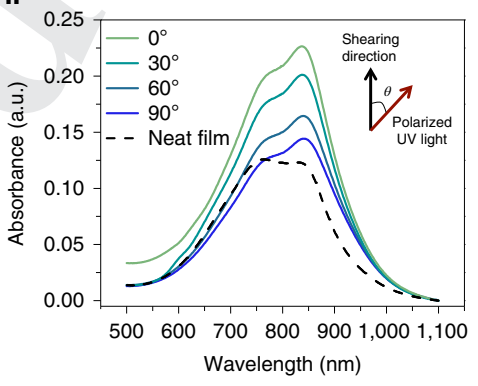

Fig. 1 | Achieving multiple length scale ordering of conjugated polymers in stretchable semiconductors through a combination of the patternedblade solution-shearing method and the nanoconfinement effect. $\mathbf{a}$, Three-dimensional schematic representing alignment of polymer semiconductor nanofibres through the solution-shearing method using a microtrench-patterned blade. b, Calculated three- (top) and two-dimensional (bottom) velocity fields (streamline representation) of the solution between the coating blade and the substrate. The simulated printing speed is $3 \mathrm{~mm} \mathrm{~s}^{-1}$. c, Scanning electron microscopy image of a microtrench-patterned blade. The height and width of the trench are $20 \mu \mathrm{m}$ and $5 \mu \mathrm{m}$, respectively. The gap between the repeating units is $10 \mu \mathrm{m}$. d, Chemical structures of the semiconducting polymer DPPDTSE and the SEBS elastomer (weight ratio: 12/88). e, Polarized optical microscopy images of an SS-neat DPPDTSE film and an SS-CONPHINE film (trench). The orientation of the cross-polarizers is shown as arrows, and the white arrows indicate the coating direction. $\mathbf{f}$, AFM phase images of the bottom surfaces of an SC-CONPHINE film and an SS-CONPHINE film (trench). g, GIXD images (left) of the SS-CONPHINE film (trench), with the incident beam oriented parallel $\left(0^{\circ}\right)$ and perpendicular $\left(90^{\circ}\right)$ to the coating direction. The dashed boxes highlight the in-plane $\pi-\pi$ stacking peaks. The right-hand plot is the intensity line cuts for the SS-CONPHINE film (trench)

Qs along the $Q_{x y}$ axis, with the incident beam oriented at different angles with respect to the shearing direction. h, Absorption spectra of polarized ultravioletvisible spectroscopy from SS-CONPHINE film (trench) (solution concentration $30 \mathrm{mg} \mathrm{ml}^{-1}$, substrate temperature $50^{\circ} \mathrm{C}^{\circ}$, blade movement speed $3 \mathrm{~mm} \mathrm{~s}^{-1}$ ). The inset shows the angles between the shearing direction and the axis of the polarizer. The dashed curve is the absorption spectra of the SC-neat DPPDTSE film with the intensity normalized for comparison. i, Optical microscope images of a neat semiconductor film and an SS-CONPHINE film under $100 \%$ strain along the shearing direction; j, AFM phase images showing the nanoscale morphology of the SS-CONPHINE films under $100 \%$ strain both parallel and perpendicular to the shearing direction. 
mobility of $1.5 \mathrm{~cm}^{2} \mathrm{~V} \mathrm{~s}^{-1}$, which changed minimally under stretching up to $100 \%$ strain. Furthermore, we extended this method to rollto-roll printing for the large-scale fabrication of stretchable semiconducting films with desired chain alignment and therefore high performance.

Achieving multi-scale morphological ordering together with mechanical stretchability in a solution-printed semiconducting film relies on two key factors: (1) generating a strong unidirectional flow field for alignment of polymer chains, and (2) 'freezing' the polymer alignment while achieving good stretchability. Inspired by the microchannel design for DNA stretching ${ }^{17}$, a coating blade with micro-trenches was designed to effectively generate an intensive unidirectional flow field to align and elongate polymer chains in the solution beneath the blade (Fig. 1b,c; Supplementary Figs. 1 and 2 describe in more detail the simulation results and rationales for the blade design). However, it remains challenging to prevent chain relaxation from the extended conformation and aligned orientation during film drying. Although this challenge has been conquered by pre-engineering the substrates into specific designs (for example nanogrooves ${ }^{18}$, dynamic templates ${ }^{19}$ ) or directional coating of pregrown nanowires/nanofibres ${ }^{20,21}$, the rough morphology and/ or high crystallinity of the resulting films are unfavourable for realizing high stretchability. Here, we hypothesize that, during the drying process, conjugated-polymer nanofibril structures formed from conjugated-polymer/elastomer phase separation may confine the conjugated-polymer chains with an extended chain conformation. Moreover, the relaxation of these larger aligned nanofibres in the elastomer matrix when compared with that of a single polymer chain should be much slower kinetically at timescales relevant to drying. Additionally, the nanoconfinement introduced during this shear coating deposition may achieve improved stretchability ${ }^{10}$. Indeed, we show that, under optimized solution-shearing conditions, semiconducting polymer films with aligned nanoconfined morphology could be fabricated through the conjugated-polymer/elastomer phase-separation-induced elasticity (termed CONPHINE) methodology, which achieves morphological ordering at multiple length scales in a single coating step. Moreover, the enhanced chain dynamics and the suppressed crystallization from the nanoconfinement effect serve to greatly improve the stretchability of the conjugated polymers ${ }^{10}$.

\section{Achieving multi-scale ordering}

A high-mobility semiconducting polymer, poly-[2,5-bis(7-decyInonadecyl)pyrrolo[3,4-c]pyrrole-1,4-(2 H,5 H)-dione-(E)-(1,2bis(5-(thiophen-2-yl)selenophen-2-yl)ethene) (DPPDTSE) ${ }^{22}$, and a soft elastomer, polystyrene-block-poly(ethylene-ran-butylene)block-polystyrene (SEBS), were chosen as the two components in the CONPHINE film to demonstrate the concept (Fig. 1d). With a lower weight percentage of DPPDTSE, thinner nanofibres were obtained in the SC-CONPHINE film, reaching a fibre diameter around $30 \mathrm{~nm}$ at $30 \mathrm{wt} \%$ DPPDTSE as shown by atomic force microscopy (AFM) phase images (Supplementary Fig. 3). Previous work found that conjugated-polymer chains can be confined along the axis of the nanofibres with such dimensions ${ }^{23}$. In order to maximize the macroscopic alignment in the CONPHINE films, it is crucial to use high shear stress to prealign the polymer chains before drying and to minimize the drying time to 'freeze in' the aligned orientation of the phase-separated nanofibrils. On the basis of these requirements, we optimized the solution-shearing process by altering the following parameters: solution concentration (Supplementary Fig. 4), shear speed (Supplementary Fig. 5) and temperature (Supplementary Fig. 6). The optimal conditions were determined to be $30 \mathrm{mg} \mathrm{ml}^{-1}, 3 \mathrm{~mm} \mathrm{~s}^{-1}$ and $50^{\circ} \mathrm{C}$ by characterization of the alignment through dichroic ratios (that is the ratio between the absorption intensity parallel to the alignment direction and that perpendicular to the alignment direction using polarized ultravio- let-visible absorption spectroscopy, Fig. 1h). Detailed analysis is provided in Supplementary Section 2. During solution shearing, the phase-separation process during the drying of the conjugated-polymer/elastomer solution is complicated ${ }^{24}$, as it is influenced not only by the Flory-Huggins interaction parameters, solubility and surface energy, but also by the crystallization and aggregation kinetics. Understanding of the process will provide insights towards further improvement of both charge transport and mechanical properties. However, it is beyond the scope of the current study.

We first provide an in-depth morphological characterization of the optimized solution-sheared (SS)-CONPHINE film (solution concentration $30 \mathrm{mg} \mathrm{ml}^{-1}$, sheared at $50^{\circ} \mathrm{C}$ with a speed of $3 \mathrm{~mm} \mathrm{~s}^{-1}$ by the microtrench blade) and clearly demonstrate the achieved multi-scale ordering. Cross-polarized optical microscopy, $\mathrm{X}$-ray photoelectron spectroscopy (XPS) and AFM characterization were employed to investigate the details of the film morphology, particularly qualitative long-range ordering at the macroscale. Cross-polarized optical microscopy showed nearly complete light extinction and uniform light re-emergence on rotation of the SS-CONPHINE film with respect to the polarizer from $0^{\circ}$ to $45^{\circ}$ to the shearing direction, indicating that long-range ordering of the polymer backbones along the coating direction was achieved (Fig. 1e). However, in the SS-neat DPPDTSE film, which does not have nanoconfined fibres (Supplementary Fig. 7), there was no clear optical anisotropy observed (Fig. 1e). As revealed by XPS, the SS-CONPHINE film formed a trilayer structure with the conjugated polymer mainly occupying the top and bottom interfaces (Supplementary Fig. 8). From AFM phase images, aligned nanofibres along the shearing direction were observed on the bottom interface of the SS-CONPHINE film (Fig. 1f), while the SC-CONPHINE film exhibited a randomly oriented nanofibre network. The nanofibres on the top surface of the SS-CONPHINE film exhibited slightly less ordering (Supplementary Fig. 8B), which may be attributed to the solvent-evaporation-induced flows on the surface of the liquid layer. At the meso- to molecular scales, grazing incidence X-ray diffraction (GIXD) measurements were applied to investigate the ordering of the crystalline regions, and ultraviolet-visible absorption spectra were used to characterize the level of short-range ordering (aggregation) and the conformation of the DPPDTSE polymer chain in aligned nanofibres. From the GIXD measurements (Fig. 1g), a significantly more intense edge-on $\pi-\pi$ stacking peak along the film coating direction parallel to the incident beam was observed in the SS-CONPHINE film when compared with the perpendicular direction, indicating the orientational order of the crystalline regions. However, this morphological anisotropy was not observed in either the SS-neat DPPDTSE film or the SC-CONPHINE film (Supplementary Figs. 9 and 10). The ultraviolet-visible spectra of the SS-CONPHINE films showed a significant increase in the ratio between the $0-0 / 0-1$ peaks and a clear redshift of the $0-0$ peak from $830 \mathrm{~nm}$ to $840 \mathrm{~nm}$ (Fig. 1h), as compared with the neat DPPDTSE film. This indicates an increase in short-range ordering (aggregation), as well as possibly improved chain planarization and linearity under one-dimensional confinement. Additionally, a maximum absorption intensity was observed when the polarization of the incident light was parallel to the coating direction, which is the same as the alignment direction for the observed nanofibres (Fig. 1h). Since the transition dipole moments for most donor-acceptor conjugated polymers tend to be oriented parallel to the polymer backbone ${ }^{25,26}$, this measured optical anisotropy reveals that the DPPDTSE polymer chain is aligned along the nanofibre in the SS-CONPHINE film, which is consistent with the observations from GIXD that the $\pi-\pi$ stacking direction orientated perpendicularly to the alignment direction of the nanofibres.

The other important benefit of nanoconfinement, in this anisotropically aligned nanofibre morphology, is the substantially enhanced stretchability of DPPDTSE owing to enhanced chain 


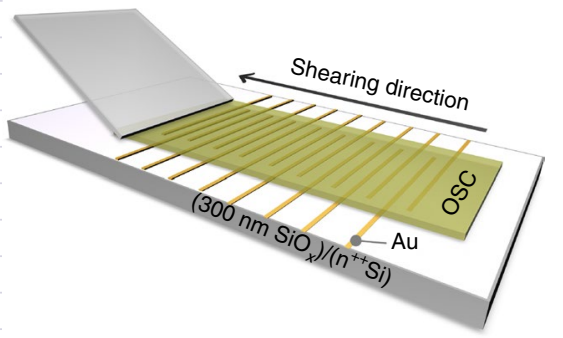

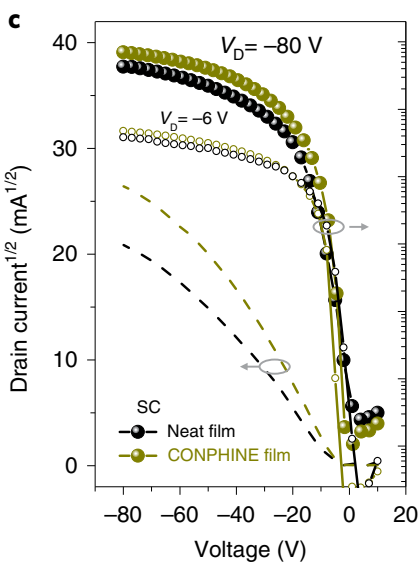

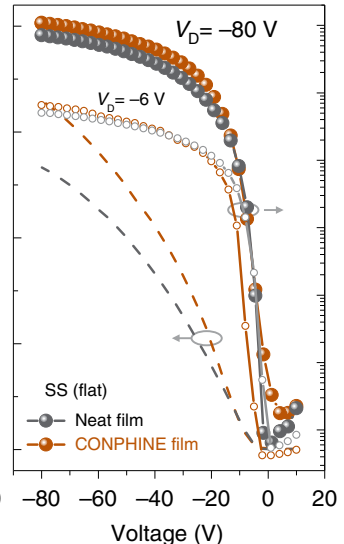

b
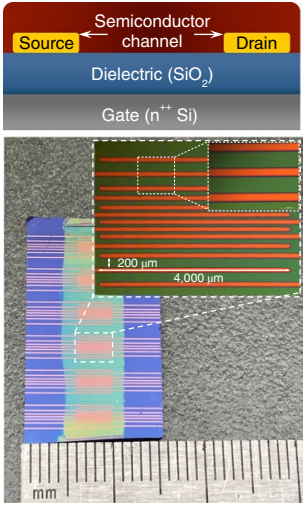

d

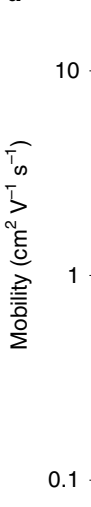

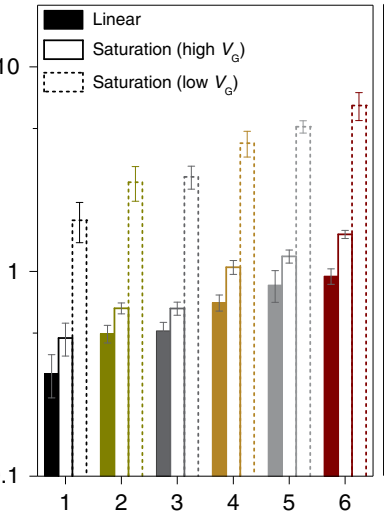
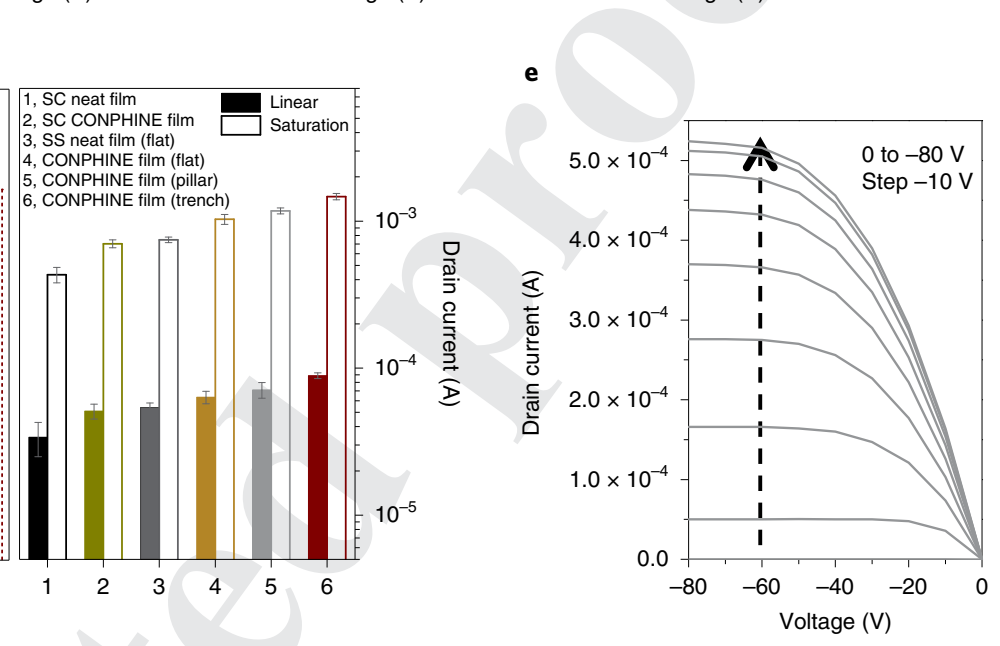

Fig. 2 | Characterization of the electrical performance of the semiconducting films fabricated using different processes. a, Schematic of the solution shearing of films on a doped $\mathrm{Si}$ substrate with $\mathrm{SiO}_{2}$ dielectric $(300 \mathrm{~nm}$ ) and gold electrodes on top as drain and source electrodes to characterize the electrical performance in TFT devices. b, Optical image and schematic diagram of bottom-gate, bottom-contact organic TFT devices (channel length $200 \mu \mathrm{m}$; channel width $4 \mathrm{~mm}$; dielectric capacitance $11.5 \mathrm{nF} \mathrm{cm}{ }^{-2}$ ). c, Transfer curves (filled symbols, with the drain voltage $V_{D}=-80 V_{\text {; }}$ dashed lines, the square root of the drain current with $V_{D}=-80 \mathrm{~V}$; open symbols, with $V_{D}=-6 \mathrm{~V}$ ) from TFTs with different semiconducting films. d, Average mobilities (estimated from the linear regime and the saturation in both high- and low-gate-voltage regimes with the average reliability factor values ${ }^{28}$ of $r_{\text {lin }}=85 \%$, $r_{\text {sat,high }}=147 \%$ and $r_{\text {sat,low }}=32 \%$, respectively) and drain currents of these different semiconducting films. The error bars represent the standard deviation obtained from six measurements. e, A typical output characteristic from a device fabricated using an SS-CONPHINE film coated with a microtrench-patterned blade.

dynamics and suppressed large crystal growth of the conjugated polymers $^{10}$. Additionally, crack propagation was inhibited by the deformable interfaces formed with the elastomer SEBS matrix ${ }^{10}$. Figure 1i shows that the aligned CONPHINE film can be stretched to $100 \%$ strain in the directions both parallel and perpendicular to the shearing direction without resulting in any cracks even at the nanoscale as characterized by AFM (Fig. 1j), while the neat film showed large cracks (width around $10 \mu \mathrm{m}$ ) at $100 \%$ strain.

\section{Transistor performance}

With both improved conformational order and morphological alignment, organic thin-film transistors (TFTs)fabricated using our SS-CONPHINE films exhibited significantly improved performance in the initial strain-free state. The semiconducting films were directly deposited (SS or SC) on top of a rigid device stack ( $40 \mathrm{~nm}$ $\mathrm{Au}$ as source and drain electrodes on octadecyltrichlorosilane (OTS)-modified $300 \mathrm{~nm} \mathrm{SiO} / \mathrm{Si}$, Fig. 2a,b) to achieve the bottomgate, bottom-contact device structure. A typical transfer curve in the saturation regime exhibited a double-slope characteristic with a downward bend at higher gate voltages, which is likely to arise from contact resistance effects (Supplementary Fig. 11) and carrier trapping ${ }^{27-30}$. Correspondingly, we calculated saturation mobility values in regions of both low and high gate voltage $\left(V_{\mathrm{G}}\right.$, Supplementary Fig. 12A). Linear-regime mobility is also listed since it is less prone to erroneous mobility extraction ${ }^{28}$. To understand how the changes in the observed morphology impact the transistor performance, we first compared the transistor characteristics of the SC-neat DPPDTSE film $(\sim 130 \mathrm{~nm})$ with those of the SC-CONPHINE film. Both the on-currents and estimated charge carrier mobilities from the SC-CONPHINE films using three methods (that is, saturation mobility in the high- $V_{\mathrm{G}}$ regime, saturation mobility in the low- $V_{\mathrm{G}}$ regime and linear-regime mobility) consistently increased by around $50 \%$ when compared with those of the neat films (Fig. 2c(left),d), suggesting that the extended DPPDTSE chain conformation and promoted DPPDTSE aggregation from spatial nanoconfinement are beneficial for charge transport. Next, the transistor characteristics of the SS-CONPHINE films were investigated. From the CONPHINE film sheared just using an unstructured (flat) blade, significantly higher on-current and charge carrier mobilities were already observed when compared with the SS-neat film (flat), indicating that polymer chain alignment improved the charge transport in the nanoconfined morphology (Fig. $2 c($ middle),d). As a comparison, 

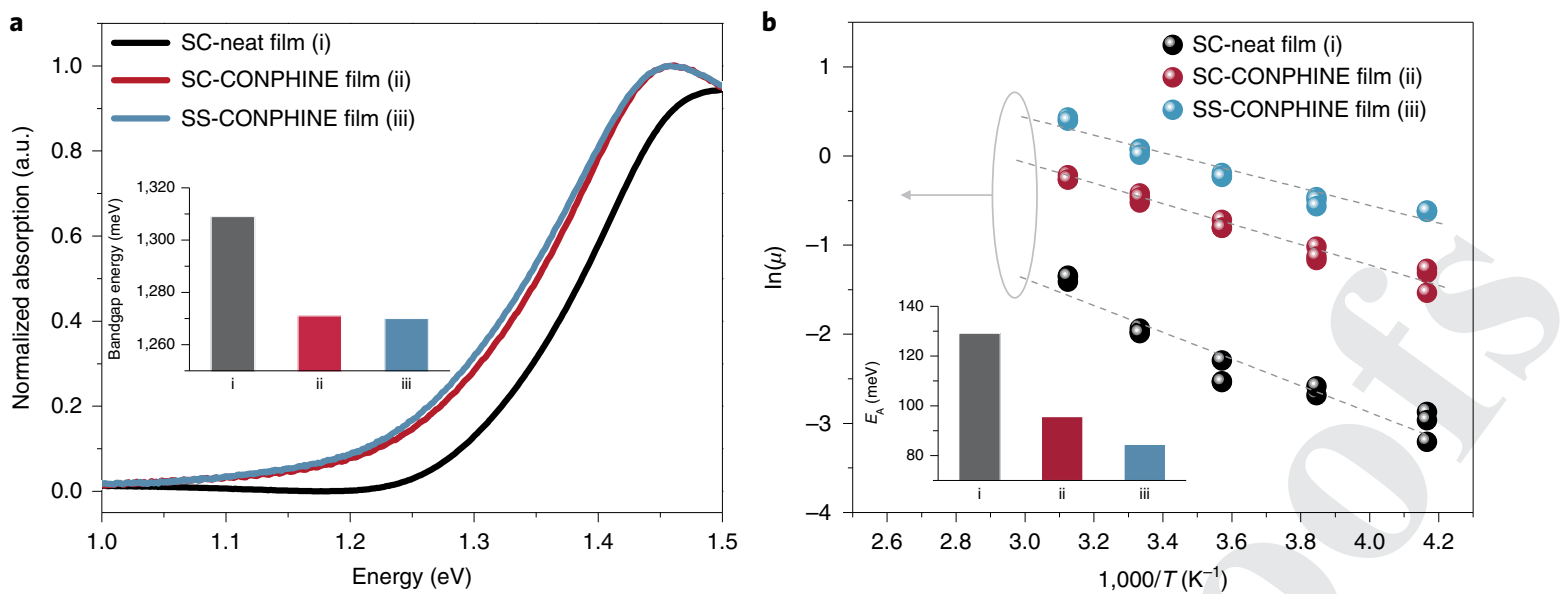

Fig. 3 Measurements of bandgap energies and activation energies for charge transport. a, Normalized UV absorption of SC-neat, SC-CONPHINE and SS-CONPHINE films (trench). The inset shows the bandgap energies of these three films. $\mathbf{b}$, Arrhenius plots of the temperature-dependent $\mu$ values for SC-neat, SC-CONPHINE and SS-CONPHINE films (trench) with a drain-source voltage $\left(V_{D S}\right)$ value of $-6 \mathrm{~V}$. The inset shows the activation energies of these three films.
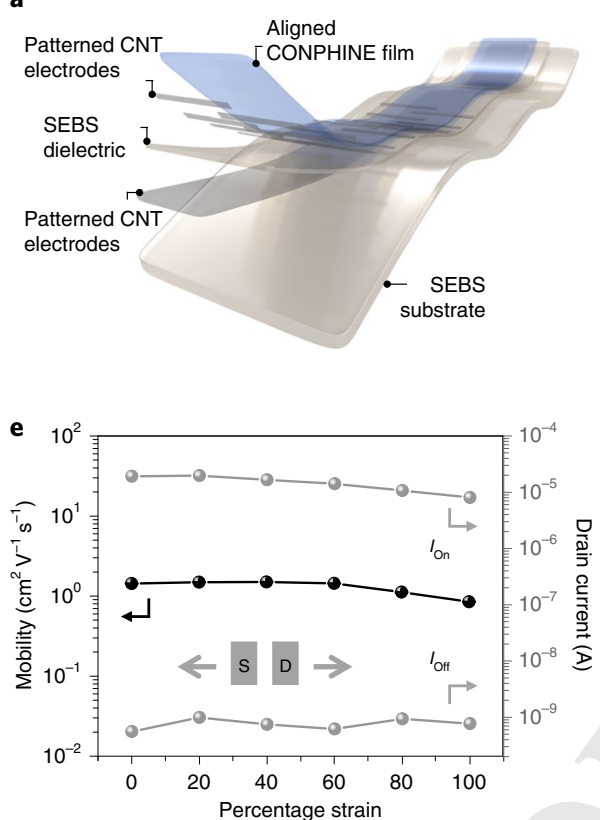

b

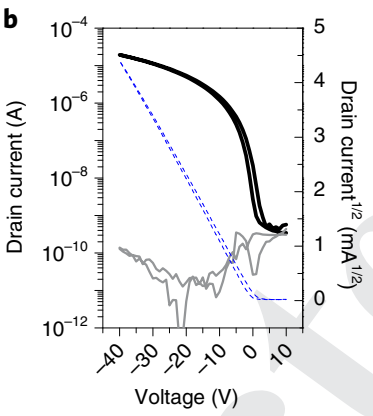

f

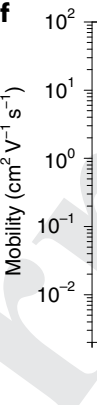

C

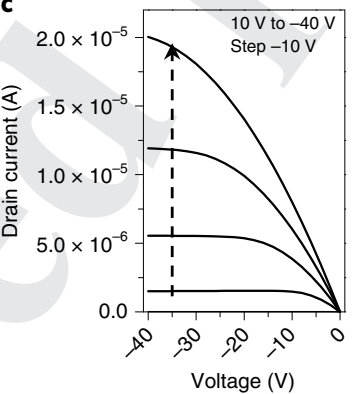

d

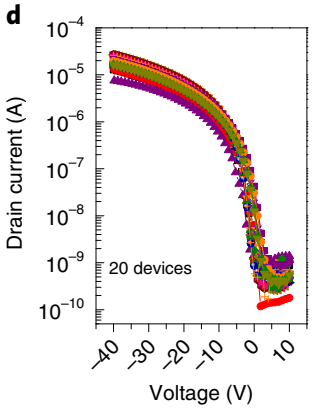

Fig. 4 | Fully stretchable transistors fabricated from the SS-CONPHINE film. a, The device structure (Supplementary Fig. 20; channel length 200 $\mu$ m; channel width $4 \mathrm{~mm}$; dielectric capacitance $\left.1.5 \mathrm{nF} \mathrm{cm}^{-2}\right)$. b. A typical transfer curve $\left(V_{D}=-40 \mathrm{~V}\right)$ at $0 \%$ strain (dashed lines, square root of the drain current; grey lines, gate current). c, A typical output characteristic at $0 \%$ strain. $\mathbf{d}$, Transfer curves from 20 devices in arrays of fully stretchable transistors at $0 \%$ strain. e,f, Changes in the on-current and saturation mobility (calculated with measured device geometry and dielectric capacitance under strain, Supplementary Table 2, Supplementary Fig. 12C) during stretching to 100\% strain, both parallel (e) and perpendicular to (f) the charge transport direction. g, Changes in the mobility after multiple stretch-release cycles (up to 1,000 cycles) at 50\% strain parallel (top) and perpendicular (bottom) to the charge transport direction. The reliability factor values of the mobilities are provided in Supplementary Table 3.

in SS-DPPDTSE neat films, less improvement was observed due to the low degree of alignment without spatial nanoconfinement. Finally, with the structured shearing blades (micropillar and microtrench shaped) at the optimized shearing conditions, the transistor performance was further improved, which can be attributed to the enhanced degree of alignment (Supplementary Fig. 1E) on the basis of the positive correlation observed between alignment and on-currents (Supplementary Fig. 13). Using the microtrenchstructured blade to fabricate SS-CONPHINE resulted in the highest on-current and linear mobility, which was around twice the linear mobility of the SC-CONPHINE film and three times the linear mobility of the SC-neat DPPDTSE film (Fig. 2c(right),d). Figure 2e shows a typical output characteristic from the device fabricated using an SS-CONPHINE film with a microtrench-patterned blade.

To evaluate the generality of this approach on different conjugated polymers for fabricating high-performance stretchable semiconductors, it was further applied to four other conjugated polymers (Supplementary Fig. 14). Highly aligned nanoconfined 


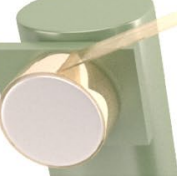

Ink
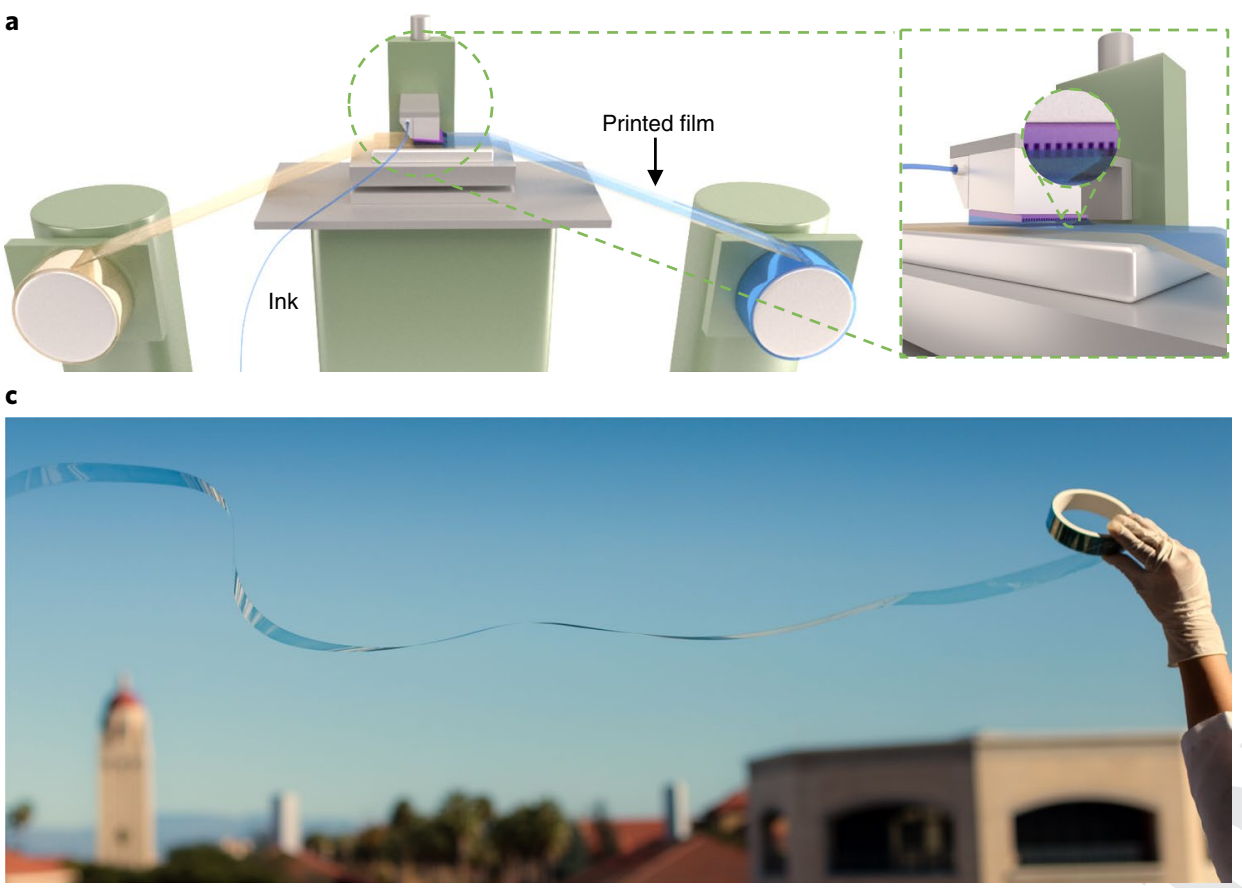

b
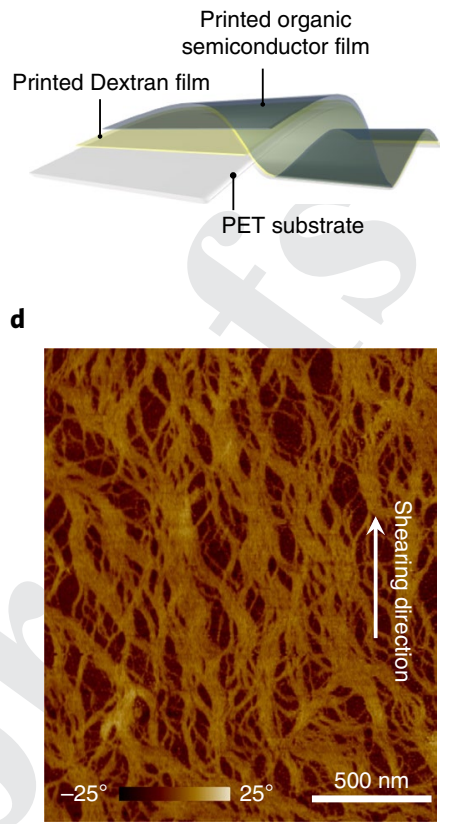

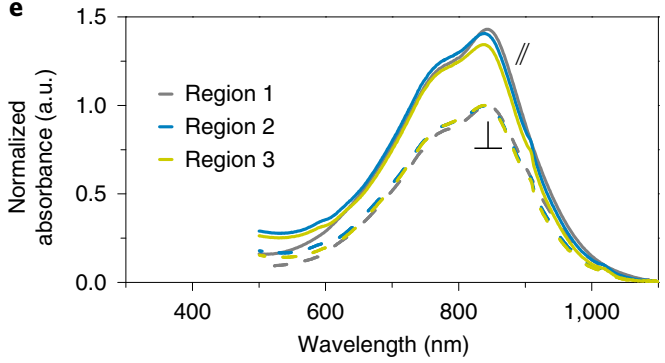

f

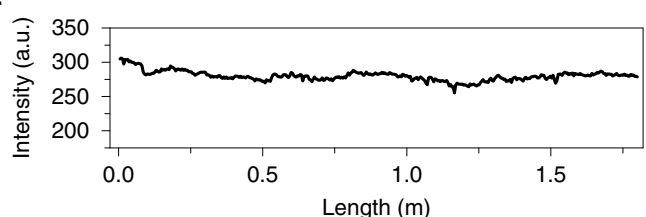

g

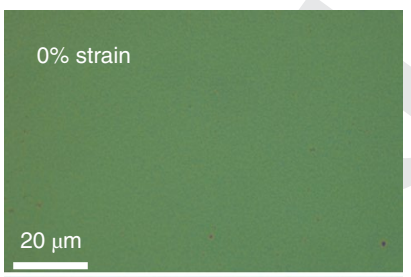

$100 \%$ strain

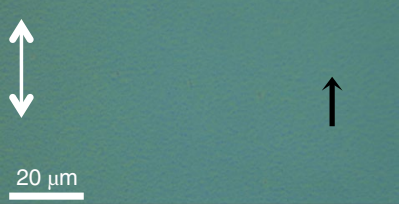

h

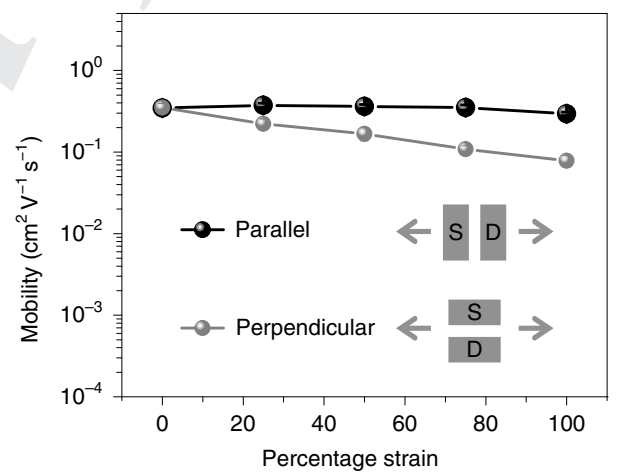

Fig. 5 | Large-area roll-to-roll coating of aligned, stretchable CONPHINE film. a, Roll-to-roll coating using a combination of a slot die coater and a microtrench-structured shearing blade. Right, magnified view of the coater setup. b, Roll-to-roll-coated film structure (PET substrate $30 \mu$ m; Dextran film $200 \mathrm{~nm}$; printed film $100 \mathrm{~nm}$ ). c, Roll of metre-scale, roll-to-roll-coated CONPHINE films on PET. d, AFM phase image of the top surface of a roll-to-rollcoated CONPHINE film. e, Polarized ultraviolet-visible absorption spectra of roll-to-roll-coated CONPHINE films from three different regions. The sign \|I $(\perp)$ denotes the film orientation when the coating direction is parallel (perpendicualr) to the axis of the polarizer. $\mathbf{f}$, Plot of the transmitted light intensity as a function of time during film coating. $\mathbf{g}$, Optical microscope images of a roll-to-roll-coated CONPHINE film under 0\% (top) and 100\% (bottom) strain. The black arrow indicates the coating direction. h, Changes in the mobility (calculated with measured device geometry and dielectric capacitance under strain $^{10}$ ) of the roll-to-roll-coated CONPHINE film with strain up to $100 \%$, both parallel and perpendicular to the charge transport direction in the fabricated fully stretchable transistors (Supplementary Fig. 20; channel length $200 \mu \mathrm{m}$; channel width $4 \mathrm{~mm}$; dielectric capacitance $1.5 \mathrm{nF}$ cm ${ }^{-2}$ ). The reliability factor values of the mobilities are provided in Supplementary Table 5.

morphologies were again achieved from all of these polymers, with their stretchability greatly improved (no cracks observed up to $100 \%$ strain, as shown in Supplementary Fig. 15). Benefiting from such multi-scale morphological ordering, the on-currents from these SS films exceed those of their SC counterparts by around two- to sixfold (Supplementary Fig. 16).

\section{Understanding the morphology-transport relationship}

To understand the link between the observed morphology and improved transistor performance, we further investigated the bandgap and transport activation energy $\left(E_{\mathrm{A}}\right)$ of the SC-neat,
SC-CONPHINE and SS-CONPHINE films. As an important parameter representing the energetic disorder in conjugated polymers due to variations in backbone conformation and aggregation states, the optical and electrochemical bandgap energies of DPPDTSE in these three films were extracted from ultraviolet-visible spectra (Fig. 3a) and cyclic voltammetry measurements (Supplementary Fig. 17), respectively. When compared with the neat film, DPPDTSE under spatial nanoconfinement exhibited a significantly lower bandgap energy (Fig. 3a and Supplementary Fig. 17), indicating better $\pi$-electron delocalization. This can be explained by the increased effective conjugation and/or more short-range aggregation due to 
the extended chain conformation under confinement. However, little difference between the spectra of SC- and SS-CONPHINE films was observed, indicating that the macroscale alignment of nanofibres did not change the bandgap energy. In order to more directly reflect the influence on charge carrier mobility, we next measured the $E_{\mathrm{A}}$ of charge transport in these semiconducting films ${ }^{31}$. The $E_{\mathrm{A}}$ can be extracted from temperature-dependent mobility $(\mu)$ measurements using the Arrhenius relation, $\mu \propto \exp \left(-E_{\mathrm{A}} / k T\right)$, where $k$ is the Boltzmann constant and $\mu$ values were measured in the linear regime under low drain-source voltage (Supplementary Fig. 18). As shown in Fig. 3b, the SC-CONPHINE film exhibited a significantly lower $E_{\mathrm{A}}$ value than the neat film, which serves as one of the explanations for the higher observed charge carrier mobility. Moreover, in the SS-CONPHINE films (fabricated with flat-, pillar- and trenchstructured blades, Supplementary Fig. 19), the alignment of nanofibres achieved may further reduce the morphological boundaries that are unfavourable for charge transport, leading to the lowest $E_{\mathrm{A}}$ and therefore the highest charge carrier mobility in the best aligned SS-CONPHINE film (trench blade).

\section{Electrical performance of stretchable transistors}

For evaluation of the electrical performance under strain, the SS-CONPHINE film was used to fabricate fully stretchable transistors in the bottom-gate, bottom-contact configuration, with carbon nanotube (CNT) networks defining the charge transport channels along the shearing direction, and SEBS as both the dielectric layer and the stretchable substrate (Fig. 4a). The fully stretchable TFT exhibits nearly ideal transistor characteristics, reaching an average mobility of $1.50 \mathrm{~cm}^{2} \mathrm{~V}^{-1} \mathrm{~s}^{-1}\left(r_{\text {sat }}=84 \%\right.$, Supplementary Fig. 21$)$ from 20 devices with relatively small variance (Fig. $4 \mathrm{~b}-\mathrm{d}$ ). Here, the lower mobilities when compared with those from semiconducting films directly deposited on $\mathrm{Si} / \mathrm{SiO}_{2}$ are due to deteriorated interface quality caused by the film transfer process during fabrication, as well as a higher contact resistance from CNT electrodes when compared with $\mathrm{Au}^{10,19}$. When stretched to $100 \%$ strain along the charge transport direction, the mobility remains almost the same (Fig. 4e and Supplementary Fig. 22A), with only a slight drop in the on-current as a result of the increased channel length under stretching. When the stretching is perpendicular to the charge transport direction, the combined effect of the slight decrease in mobility and the device geometric change makes the transfer curve very stable under strain up to $100 \%$ (Fig. $4 \mathrm{f}$ and Supplementary Fig. 22B). Furthermore, this fully stretchable TFT exhibits a good robustness with stable device performance over 1,000 repeated stretching cycles (at four cycles per second) to $50 \%$ strain (Fig. $4 \mathrm{~g}$ and Supplementary Figs. $22 \mathrm{C}$ and D). As a comparison, the strain effects on the mobilities ( $r_{\text {sat }}$ provided in Supplementary Table 4) from the SC-neat DPPDTSE film and SS-CONPHINE film are provided in Supplementary Figs. 23 and 24 , respectively.

\section{Large-area roll-to-roll fabrication of aligned CONPHINE film}

We further extended this method for multi-scale morphology control to an industrially relevant roll-to-roll coating process to fabricate high-performance, stretchable semiconducting films metres in length, with a high coating speed of $1 / 6 \mathrm{~m} \mathrm{~min}^{-1}$ (Fig. 5a,c, Supplementary Movie 1). The consumption of conjugated polymer is estimated to be only about $0.02 \mathrm{~g} \mathrm{~m}^{-2}$. A polyethylene terephthalate (PET) roll was used as the printing substrate. In order to release the printed semiconducting film from the PET substrate for electrical and mechanical testing, a water-soluble sacrificial layer (Dextran) was printed prior to the printing of the aligned CONPHINE film (Fig. 5b). As revealed by AFM phase images and XPS characterization, the print-aligned CONPHINE film showed a bilayer structure with the aligned network of nanofibril conjugated polymer on its top surface (Fig. $5 \mathrm{~d}$ and Supplementary Fig. 25). The uniformity of the printed film was examined using ultraviolet-visible absorption spectroscopy and AFM on three randomly chosen regions, all of which had similar spectra with nearly identical dichroic ratios and similar morphologies (Fig. 5d,e and Supplementary Fig. 26). On the large scale, as characterized by the absorption intensity of the film, the thickness variation over the metre-long continuously coated film was less than $8 \%$ (Fig. $5 \mathrm{f}$ and Supplementary Fig. 27). This printed CONPHINE film also exhibited both high stretchability and good electrical performance (Fig. 5 g,h, Supplementary Fig. 28 and Table 5). Compared with that of the small-area SS-CONPHINE film, the reduction in electrical performance here is attributed to the lower degree of overall alignment (the dichroic ratio is 1.4 here as compared with 1.7 from the small-area SS-CONPHINE film) and the rougher surface of the PET substrate when compared with $\mathrm{Si} / \mathrm{SiO}_{x}$ (with averaged surface roughness of $9.5 \mathrm{~nm}$ versus $1.1 \mathrm{~nm}$, Supplementary Fig. 29). Nevertheless, when compared with other reported semiconducting films fabricated by roll-to-roll printing, the performance of the printed aligned CONPHINE film is still among the highest (Supplementary Table 6).

In summary, the physical nanoconfinement effect and SS fabrication with a patterned microtrench blade were used to simultaneously achieve both enhanced chain dynamics ${ }^{10}$ and multi-scale morphological order for conjugated polymers, and therefore imparted greatly improved stretchability as well as significantly enhanced electrical performance. This processing approach is expected to benefit the development of stretchable semiconductors towards high-performance, low-cost and mass-produced stretchable skin-like electronics.

\section{Online content}

Any methods, additional references, Nature Research reporting summaries, source data, extended data, supplementary information, acknowledgements, peer review information; details of author contributions and competing interests; and statements of data and code availability are available at https://doi.org/10.1038/s41563019-0340-5.

Received: 15 July 2018; Accepted: 12 March 2019;

\section{References}

1. Chu, B., Burnett, W., Chung, J. W. \& Bao, Z. Bring on the bodyNET. Nat. News 549, 328-330 (2017).

2. Wang, S., Oh, J. Y., Xu, J., Tran, H. \& Bao, Z. Skin-inspired electronics: an emerging paradigm. Acc. Chem. Res. 51, 1033-1045 (2018).

3. Someya, T., Bao, Z. \& Malliaras, G. G. The rise of plastic bioelectronics. Nature 540, 379-385 (2016).

4. Wang, S. et al. Skin electronics from scalable fabrication of an intrinsically stretchable transistor array. Nature 555, 83-88 (2018).

5. Wang, N. G. J., Gasperini, A. \& Bao, Z. Stretchable polymer semiconductors for plastic electronics. Adv. Electron. Mater. 4, 1700429 (2018).

6. Root, S. E., Savagatrup, S., Printz, A. D., Rodriquez, D. \& Lipomi, D. J. Mechanical properties of organic semiconductors for stretchable, highly flexible, and mechanically robust electronics. Chem. Rev. 117, 6467-6499 (2017).

7. International Technology Roadmap for Semiconductors (ITRS, 2012)

8. Oh, J. Y. et al. Intrinsically stretchable and healable semiconducting polymer for organic transistors. Nature 539, 411-415 (2016).

9. Müller, C. et al. Tough, semiconducting polyethylene-poly(3-hexylthiophene) diblock copolymers. Adv. Funct. Mater. 17, 2674-2679 (2007).

10. $\mathrm{Xu}, \mathrm{J}$. et al. Highly stretchable polymer semiconductor films through the nanoconfinement effect. Science 355, 59-64 (2017).

11. Napolitano, S., Glynos, E. \& Tito, N. B. Glass transition of polymers in bulk, confined geometries, and near interfaces. Rep. Prog. Phys. 80, 036602 (2017).

12. Lee, Y. et al. Deformable organic nanowire field-effect transistors. Adv. Mater. 30, 1704401 (2018).

13. Eunjoo, S. et al. Stretchable and transparent organic semiconducting thin film with conjugated polymer nanowires embedded in an elastomeric matrix. Adv. Electron. Mater. 2, 1500250 (2016).

14. Venkateshvaran, D. et al. Approaching disorder-free transport in highmobility conjugated polymers. Nature 515, 384-388 (2014). 
15. Jackson, N. E. et al. Conformational order in aggregates of conjugated polymers. J. Am. Chem. Soc. 137, 6254-6262 (2015).

16. Wang, G. et al. Aggregation control in natural brush-printed conjugated polymer films and implications for enhancing charge transport. Proc. Natl Acad. Sci. USA 114, E10066-E10073 (2017).

17. Dai, L., Renner, C. B. \& Doyle, P. S. The polymer physics of single DNA confined in nanochannels. Adv. Colloid. Interface Sci. 232, 80-100 (2016).

18. Tseng, H.-R. et al. High mobility field effect transistors based on macroscopically oriented regioregular copolymers. Nano Lett. 12, 6353-6357 (2012).

19. Mohammadi, E. et al. Dynamic-template-directed multiscale assembly for large-area coating of highly-aligned conjugated polymer thin films. Nat. Commun. 8, 16070 (2017).

20. Persson, N. E., Chu, P.-H., McBride, M., Grover, M. \& Reichmanis, E. Nucleation, growth, and alignment of poly(3-hexylthiophene) nanofibers for high-performance OFETs. Acc. Chem. Res. 50, 932-942 (2017).

21. Chang, M., Su, Z. \& Egap, E. Alignment and charge transport of onedimensional conjugated polymer nanowires in insulating polymer blends. Macromolecules 49, 9449-9456 (2016).

22. Kang, I., Yun, H.-J., Chung, D. S., Kwon, S.-K. \& Kim, Y.-H. Record high hole mobility in polymer semiconductors via side-chain engineering. J. Am. Chem. Soc. 135, 14896-14899 (2013).

23. Zheng, Z. et al. Uniaxial alignment of liquid-crystalline conjugated polymers by nanoconfinement. Nano Lett. 7, 987-992 (2007)

24. Zhang, G. et al. Versatile interpenetrating polymer network approach to robust stretchable electronic devices. Chem. Mater. 29, 7645-7652 (2017).

25 . Schott, S. et al. Charge-transport anisotropy in a uniaxially aligned diketopyrrolopyrrole-based copolymer. Adv. Mater. 27, 7356-7364 (2015).

26. Shaw, L. et al. Direct uniaxial alignment of a donor-acceptor semiconducting polymer using single-step solution shearing. ACS Appl. Mater. Interfaces $\mathbf{8}$, 9285-9296 (2016).

27. McCulloch, I., Salleo, A. \& Chabinyc, M. Avoid the kinks when measuring mobility. Science 352, 1521-1522 (2016).

28. Choi, H. H., Cho, K., Frisbie, C. D., Sirringhaus, H. \& Podzorov, V. Critical assessment of charge mobility extraction in FETs. Nat. Mater. 17, 2-7 (2017).

29. Bittle, E. G., Basham, J. I., Jackson, T. N., Jurchescu, O. D. \& Gundlach, D. J. Mobility overestimation due to gated contacts in organic field-effect transistors. Nat. Commun. 7, 10908 (2016).

30. Phan, H. et al. Electrical double-slope nonideality in organic field-effect transistors. Adv. Funct. Mater. 28, 1707221 (2018).
31. Nikolka, M. et al. Correlation of disorder and charge transport in a range of indacenodithiophene-based semiconducting polymers. Adv. Electron. Mater. 3, 1700410 (2017)

\section{Acknowledgements}

This work is supported by the US Department of Energy, Office of Science, Basic Energy Sciences, under award DE-SC0016523 (material characterization) and by Samsung Electronics (device fabrication and characterization). Y.-H.K. acknowledges support from the NRF Korea (2015R1A2A1A10055620). L.S. acknowledges support from the Kodak Graduate Fellowship. The GIXD measurements were made at beamlines 11-3 and 7-2 of the Stanford Synchrotron Radiation Light Source, which are supported by the Director, Office of Science, Office of Basic Energy Sciences, of the US Department of Energy. Part of this work was performed at the Stanford Nano Shared Facilities (SNSF), supported by the National Science Foundation under award ECCS-1542152.

\section{Author contributions}

J.X. and Z.B. conceived and designed the experiments; J.X., H.-C.W., A.E. and K.G. fabricated the films; J.X., H.-C.W., C.Z., A.E. and M.N. fabricated the transistor devices and made the measurements; J.X. and L.S. carried out the flow simulations; X.G., F.M.-L. and H.-C.W. did the GIXD characterizations; C.C. and V.R.F. carried out the XPS and SEM characterizations; S.W., Y.K. and Y.-Q.Z. fabricated the micro-structured blades; N.G.-J.W., T.K., Y.-H.K., and H.Y. provided the conjugated polymers; S.L., D.Z. and J.L. contributed to the initial design of the printing ink. J.W.C. and B.M. advised on the discussion of results. J.X. organized the data and wrote the first draft of the manuscript. All authors reviewed and commented on the manuscript. Z.B. directed the project.

\section{Competing interests}

The authors declare no competing interests.

\section{Additional information}

Supplementary information is available for this paper at https://doi.org/10.1038/ s41563-019-0340-5.

Reprints and permissions information is available at www.nature.com/reprints.

Correspondence and requests for materials should be addressed to Z.B.

Publisher's note: Springer Nature remains neutral with regard to jurisdictional claims in published maps and institutional affiliations.

(C) The Author(s), under exclusive licence to Springer Nature Limited 2019 


\section{Methods}

Materials. Polymer poly(tetrathienoacene-diketopyrrolopyrrole) was

provided by Corning Incorporated. The conjugated polymers DPPDTSE poly[(5,6-difluoro2,1,3-benzothiadiazol-4,7-diyl)-alt-(3,3"'-di(2-decyltetradec yl) $-2,2^{\prime} ; 5^{\prime}, 2^{\prime \prime} ; 5^{\prime \prime}, 2^{\prime \prime \prime}$-quaterthiophen-5,5"'-diyl) $]^{32}$, poly[2,5-bis(4-decyltetradecyl) pyrrolo[3,4-c]pyrrole-1,4-(2 H,5 H)-dione-(E)-1,2-di(2,2'-bithiophen-5-yl) ethene ${ }^{10}$ and poly $[(2,5$-di(5-decyl-1-pentadecyl)-3,6-di(thiophen-2-yl) pyrrolo[3,4-c]pyrrole-1,4(2 H,5 H)-dione)-alt-((E)-1,2-di(thiophen-2-yl)ethane)] ${ }^{10}$ were synthesized via reported methods. The number-averaged molecular weight, weight-averaged molecular weight and polydispersity index of these polymers were measured using high-temperature size exclusion chromatography performed at $200^{\circ} \mathrm{C}$ with 1,2,4-trichlorobenzene as the solvent on a Tosoh high-temperature EcoSEC equipped with a single TSKgel gel permeation chromatography column (GMHHR-H; $300 \mathrm{~mm} \times 7.8 \mathrm{~mm}$ ) calibrated with monodisperse polystyrene standards. The molecular weights and polydispersity indices of these polymers are shown in Supplementary Table 1. SEBS (H1221 with volume fraction of poly(ethylene-co-butylene) 88\%, H1062 with volume fraction of poly(ethyleneco-butylene) $82 \%$ and $\mathrm{H} 1052$ with volume fraction of poly(ethylene-co-butylene) $80 \%$ ) was provided by Asahi Kasei. SEBS H1221 is used in the CONPHINE film system. SEBS H1052 is only used as the dielectric layer and SEBS H1062 is used for elastomer substrate and encapsulation layer preparation in fully stretchable devices. Poly(dimethylsiloxane) (PDMS, Sylgard 184, Dow Corning) was prepared at a ratio of $12: 1$ (base:cross-linker, w:w) and cured at $70^{\circ} \mathrm{C}$ for $1 \mathrm{~h}$ for use in the lamination and as the stretching substrate. Dextran, OTS and all the solvents, such as chlorobenzene, toluene and trichloroethylene, were purchased from SigmaAldrich and used as received.

Thin-film preparation and characterization. Blade fabrication. A silicon mould was prepared first by patterning a photoresist mask with a Cr masking layer on top of a silicon wafer using standard photolithography, followed by dry ion etching (PlasmaTherm-DSE). There were three etching steps: (1) treating for $2.5 \mathrm{~s}$ with $10 \mathrm{~V}$ bias voltage at $2,000 \mathrm{~W}$ in a $\mathrm{C}_{4} \mathrm{~F}_{8}$ and $\mathrm{Ar}$ (150/30 flow speed) environment at a pressure of $25 \mathrm{mTorr}$; (2) treating for $1.5 \mathrm{~s}$ with $250 \mathrm{~V}$ bias voltage at $2,000 \mathrm{~W}$ in an $\mathrm{SF}_{6}$ and $\mathrm{Ar}$ (150/30 flow speed) environment at 40 mTorr; (3) treating for $3.0 \mathrm{~s}$ with $10 \mathrm{~V}$ bias voltage at $3,000 \mathrm{~W}$ in an $\mathrm{SF}_{6}$ and Ar mixture (300/30 flow speed) environment at $75 \mathrm{mTorr}$. The silicon was etched by $20-25 \mu \mathrm{m}$ and then acetone was used to remove the photoresist layer. The blade was then treated with oxygen plasma for $3 \mathrm{~min}$ at $150 \mathrm{~W}$ and immersed in a $0.1 \mathrm{vol} . \%$ OTS/trichloroethylene solution for $20 \mathrm{~min}$ at room temperature. After sonicating with toluene for $10 \mathrm{~min}$, the wafer was dried with nitrogen.

Substrate preparation. The bare $\mathrm{Si}$ or doped $\mathrm{Si}$ wafers with a $\mathrm{SiO}_{2}$ dielectric $(300 \mathrm{~nm})$ were treated by a method published previously to form a highly hydrophobic crystalline OTS monolayer on the surfaces ${ }^{33}$. The wafers were cut into pieces $(1.5 \mathrm{~cm} \times 3 \mathrm{~cm})$. Then a PDMS stamp $(1.2 \mathrm{~cm} \times 2.5 \mathrm{~cm})$ was laminated on the middle area of the substrate to protect the OTS layer. Oxygen plasma $\left(150 \mathrm{~W}, 200\right.$ mTorr of $\mathrm{O}_{2}$ for $\left.5 \mathrm{~s}\right)$ was used to remove the exposed OTS at the edges of the substrate in order to facilitate a more stable meniscus during coating. After removing the PDMS stamps and cleaning with toluene, the edge-etched OTS-modified substrates were ready for film shearing or electrode deposition. The modified bare Si was used as substrates for the GIXD characterization while modified $\mathrm{Si} / \mathrm{SiO}_{x}$ was used as substrates for preparing devices.

Semiconducting film preparation. The polymer solutions for the SS $\left(30 \mathrm{mg} \mathrm{ml}^{-1}\right)$ and SC $\left(10 \mathrm{mg} \mathrm{ml}^{-1}\right)$ CONPHINE semiconducting films were prepared by dissolving the conjugated polymers and SEBS at a weight ratio of 3:7 in chlorobenzene at $90^{\circ} \mathrm{C}$ for $1 \mathrm{~h}$. Neat conjugated-polymer solutions of $20 \mathrm{mg} \mathrm{ml}^{-1}$ and $10 \mathrm{mg} \mathrm{ml}^{-1}$ were used to prepare the SS- and SC-neat films. Before shearing, the substrate was placed on a heating plate $\left(50^{\circ} \mathrm{C}\right)$ with the shearing blade tilted at $8^{\circ}$ from the horizontal. Solution shearing was performed at coating speeds ranging from 0.5 to $10 \mathrm{~mm} \mathrm{~s}^{-1}$, with a gap size set the same as the height of the microstructures on the shearing blades. For the unstructured flat blades, the gap was set to $20 \mu \mathrm{m}$ (equal to the height of both pillars and trenches). A side camera was used to adjust the gap. For each shearing process, $3 \mu \mathrm{cm}^{-2}$ of solution was injected into the gap with a micropipette. The SC films were spin coated at 1,000 r.p.m. for $1 \mathrm{~min}$. Both the SS and SC films were annealed inside a nitrogen glove box at $150^{\circ} \mathrm{C}$ for $1 \mathrm{~h}$.

Morphology characterization. Optical images of the films were taken with a crosspolarized optical microscope (Leica DM4000M). The AFM images were obtained with tapping-mode AFM using a Multimode Nanoscope III (Digital Instruments/ Veeco Metrology Group). Thickness measurements were made using AFM. The thicknesses of the SS film solution sheared at $3 \mathrm{~mm} \mathrm{~s}^{-1}$ and all of the SC films are between 100 and $130 \mathrm{~nm}$. The thicknesses of SS-CONPHINE films prepared at other speeds are summarized in Supplementary Fig. 5B. Ultraviolet-visible spectra were measured with an Agilent Cary 6000i UV/vis/NIR spectroscope, which was equipped with a rotational polarizer to measure the absorption intensity with the polarization parallel and perpendicular to the shearing/stretching directions. GIXD images in Fig. $1 \mathrm{~g}$ and Supplementary Fig. 10 were collected in a helium atmosphere in the Stanford Synchrotron Radiation Light Source at beamlines 11-3 and 7-2 with beam energies of $12.7 \mathrm{keV}$ and $14 \mathrm{keV}$, respectively. The $\mathrm{X}$-ray incidence angle was $0.12^{\circ}$. Numerical integration of the diffraction peak areas was performed using WxDiff. XPS measurements were made on a PHI VersaProbe 3 XPS microprobe. Sputtering was carried out at $5 \mathrm{kV}$ and $20 \mathrm{nA}$ with an $\mathrm{Ar} 2,500+$ gas cluster ion beam gun.

Electrical characterization. The initial electrical characterization was performed on bottom-gate, bottom-contact field effect transistors fabricated by directly solution shearing (spin coating) semiconducting films on top of a rigid device stack ( $40 \mathrm{~nm}$ Au source and drain electrodes on OTS-modified $300 \mathrm{~nm} \mathrm{SiO} / 2 \mathrm{Si}$ ). The channel length and width are $200 \mu \mathrm{m}$ and $4 \mathrm{~mm}$, respectively. The capacitance of the dielectric layer is $11.5 \mathrm{nF} \mathrm{cm}^{-2}$. The fabricated devices were stored under vacuum and tested using a probe station in an ambient environment connected to a Keithley 4200 analyser.

The temperature-dependent field effect transistor characteristics of semiconducting films were measured in a cryostat. Here, the mobility was measured in the linear regime under low drain-source voltage $(-6 \mathrm{~V})$, which was assumed to yield a uniform electric field strength along the channel and minimize any effects of heating on our result ${ }^{34}$.

Fully stretchable devices were prepared as bottom-gate, bottom-contact device structures according to our previously reported method ${ }^{10}$. The channel length and width are $200 \mu \mathrm{m}$ and $4 \mathrm{~mm}$, respectively. The capacitance of the dielectric layer was measured as $1.5 \mathrm{nF} \mathrm{cm}^{-2}$. All the electrical characteristics of the devices were measured with a Keithley 4200 analyser in an ambient environment. The mobility of semiconducting films was calculated with measured device geometry and dielectric capacitance under strain (Supplementary Table 2).

Roll-to-roll fabrication and characterization. First, the PET substrate was treated with oxygen $\left(150 \mathrm{~W}, 200 \mathrm{mTorr}\right.$ of $\mathrm{O}_{2}$ for $2 \mathrm{~min}$ ) to ensure good wettability. Then a solution of $10 \mathrm{wt} \%$ Dextran in $\mathrm{H}_{2} \mathrm{O}$ was coated on the PET substrate by a continuous roll-to-roll coating process at a speed of $6 \mathrm{~mm} \mathrm{~s}^{-1}$ using a flat, unpatterned shearing blade. The gap distance here was set at around $50 \mu \mathrm{m}$. The semiconducting layer was then roll-to-roll coated on the top of the dried Dextran layer with a microtrench-structured shearing blade at a speed of $3 \mathrm{~mm} \mathrm{~s}^{-1}$. Here, the gap of the microtrench blade and the PET substrate was set to the height of the trench. The semiconductor ink $\left(25 \mathrm{mg} \mathrm{ml}^{-1}\right)$ was prepared by dissolving DPPDTSE and SEBS with a weight ratio of $3: 7$ in chlorobenzene at $90^{\circ} \mathrm{C}$ for $1 \mathrm{~h}$. The Dextran or semiconductor ink was fed through a PTFE tube with a $0.5 \mathrm{~mm}$ inner diameter by a syringe pump. The PET substrate was heated to $50^{\circ} \mathrm{C}$ during the roll-to-roll printing process. A magnetic tension controller was coupled to the feeding roll and used to control the flatness of the PET substrate. After printing, the samples were annealed at $150^{\circ} \mathrm{C}$ for $1 \mathrm{~h}$ under nitrogen. By soaking in water, the printed semiconductor layer could be easily transferred from the PET substrate to either a PDMS stamp for morphology characterization or a stretchable device stack (bottom-gate, bottom-contact device structure, source and drain electrodes CNTs, dielectric SEBS, gate electrodes CNTs) for electrical performance testing (channel length $200 \mu \mathrm{m}$; channel width $4 \mathrm{~mm}$; dielectric capacitance $1.5 \mathrm{nF} \mathrm{cm}^{-2}$ ). The mobility of the semiconducting films was calculated with measured device geometry and dielectric capacitance under strain ${ }^{10}$.

\section{Data availability}

The authors declare that all data supporting the findings of this study are available within the paper and its Supplementary Information. Other supporting data are available from the corresponding author upon request.

\section{References}

32. Liu, Y. et al. Aggregation and morphology control enables multiple cases of high-efficiency polymer solar cells. Nat. Commun. 5, 5293 (2014).

33. Chortos, A. et al. Highly stretchable transistors using a microcracked organic semiconductor. Adv. Mater. 26, 4253-4259 (2014).

34. Nikiforov, O. G. et al. Current-induced Joule heating and electrical field effects in low temperature measurements on TIPS pentacene thin film transistors. Adv. Electron. Mater. 2, 1600163 (2016). 


\section{QUERY FORM}

\begin{tabular}{|l|c|}
\hline \multicolumn{2}{|c|}{ Nature Materials } \\
\hline Manuscript ID & [Art. Id: 340] \\
\hline Author & Jie Xu \\
\hline
\end{tabular}

\section{AUTHOR:}

The following queries have arisen during the editing of your manuscript. Please answer by making the requisite corrections directly in the e.proofing tool rather than marking them up on the PDF. This will ensure that your corrections are incorporated accurately and that your paper is published as quickly as possible.

\begin{tabular}{|c|c|}
\hline Query No. & Nature of Query \\
\hline Q1: & Please confirm or correct the city/country name inserted in affiliation 6. \\
\hline Q2: & $\begin{array}{l}\text { Affiliation } 6 \text { were repited and affiliation } 9 \text { link were not provided so i have inserted after } 8 \text { so affiliation has been } \\
\text { reordered please check. }\end{array}$ \\
\hline Q3: & $\begin{array}{l}\text { Please note that we reserve 'significant' and its derivatives for statistical significance; please reword where this is } \\
\text { not the intended meaning (substantial, important, and so on). }\end{array}$ \\
\hline Q4: & $\begin{array}{l}\text { Please check your article carefully, coordinate with any co-authors and enter all final edits clearly in the eproof, } \\
\text { remembering to save frequently. Once corrections are submitted, we cannot routinely make further changes to } \\
\text { the article. }\end{array}$ \\
\hline Q5: & $\begin{array}{l}\text { Note that the eproof should be amended in only one browser window at any one time; otherwise changes will be } \\
\text { overwritten. }\end{array}$ \\
\hline Q6: & $\begin{array}{l}\text { Author surnames have been highlighted. Please check these carefully and adjust if the first name or surname is } \\
\text { marked up incorrectly. Note that changes here will affect indexing of your article in public repositories such as } \\
\text { PubMed. Also, carefully check the spelling and numbering of all author names and affiliations, and the corre- } \\
\text { sponding email address(es). }\end{array}$ \\
\hline Q7: & $\begin{array}{l}\text { Please note that after the paper has been formally accepted you can only provide amended Supplementary Infor- } \\
\text { mation files for critical changes to the scientific content, not for style. You should clearly explain what changes } \\
\text { have been made if you do resupply any such files. }\end{array}$ \\
\hline Q8: & Please provide units for intensity in fig. $1 \mathrm{~g}$. \\
\hline Q9: & Is the insertion of 'drain-source voltage' as the definition of ' $V_{\mathrm{DS}}$ ' correct? \\
\hline Q10: & Dashed lines are present in Figure $3 \mathrm{~b}$ but not mentioned in the legend. Please explain what these denote. \\
\hline Q11: & Is the insertion of 'organic semiconductor' in Fig. $5 \mathrm{~b}$ as the definition of 'OSC' correct? \\
\hline Q12: & Is the insertion of 'gel permeation chromatography' as the definition of 'GPC' correct? \\
\hline Q13: & Is the insertion of 'bottom-gate, bottom-contact' as the definition of 'BCBG' correct? \\
\hline Q14: & None of the authors listed have the initials 'C.C.' found in the author contributions. Please amend accordingly. \\
\hline Q15: & $\begin{array}{l}\text { 'G.-J.N.W.' has been changed to 'N.G.-J.W.' in the author contributions to match the author name as given. Please } \\
\text { confirm that this is correct. }\end{array}$ \\
\hline
\end{tabular}

